\title{
Dirección estratégica a través del emprendimiento y modelos de negocio
}

\author{
Strategic Direction Through Entrepreneurship and Business Models \\ Direção estratégica através do empreendedorismo e modelos de negócios \\ Gabriela Velasco Rodríguez \\ Universidad Autónoma de Ciudad Juárez, México \\ gvelasco@uacj.mx \\ https://orcid.org/0000-0003-2651-6905
}

\section{Resumen}

Este trabajo plantea una revisión bibliográfica sobre las escuelas de dirección estratégica, así como las perspectivas del emprendimiento y las propuestas de modelos de negocio que emergen en un contexto de cambios tecnológicos y de mercado. Esto con la meta de diferenciar los elementos de la dirección estratégica mediante las perspectivas que describen al emprendimiento y las características de los modelos de negocio. El método comprende una investigación básica que determina un marco teórico para alcanzar el objetivo propuesto.

Como parte de las conclusiones se menciona que las estrategias son las causas de la relación entre el emprendimiento y la innovación de los modelos de negocio, ya que la etapa de gestación del emprendimiento permite vislumbrar el tipo de modelo de negocio que se pretende establecer con base en la naturaleza de las tendencias de dirección estratégica, la cual, a su vez, ofrece un marco de referencia clave para comprender la evolución del pensamiento estratégico.

Palabras claves: administración de empresas, dirección a través de objetivos, empresario. 


\section{Abstract}

This article proposes a bibliographic review of the schools of strategic management, as well as the perspectives of entrepreneurship and the proposals of business models that emerge in a context of technological changes and markets. The general objective of this research is to differentiate the elements of strategic management through the perspectives that describe entrepreneurship and the characteristics of business models. The method comprises a basic research that determines a theoretical framework to achieve the proposed objective.

As part of the conclusions it is highlighted that the strategies are the causes of the relationship between entrepreneurship and the innovation of the business models, since the gestation stage of the enterprise allows to glimpse the type of business model that is intended to be established based on the nature of strategic management trends that provides a framework key reference to understand the evolution of strategic thinking.

Keywords: business management, management by objectives, entrepreneur.

\section{Resumo}

Este artigo propõe uma revisão bibliográfica das escolas de gestão estratégica, bem como as perspectivas do empreendedorismo e as propostas de modelos de negócios que emergem em um contexto de mudanças tecnológicas e de mercado. Isso com o objetivo de diferenciar os elementos da gestão estratégica através das perspectivas que descrevem o empreendedorismo e as características dos modelos de negócios. O método inclui uma investigação básica que determina uma estrutura teórica para alcançar o objetivo proposto.

Como parte das conclusões, menciona-se que as estratégias são as causas da relação entre empreendedorismo e inovação de modelos de negócios, uma vez que o estágio de gestação do empreendedorismo permite vislumbrar o tipo de modelo de negócio que se pretende estabelecer com base na natureza das tendências de gerenciamento estratégico, que, por sua vez, oferece um quadro de referência fundamental para a compreensão da evolução do pensamento estratégico.

Palavras-chave: administração de empresas, gestão por objetivos, empreendedor.

Fecha Recepción: Junio 2019 Fecha Aceptación: Diciembre 2019 

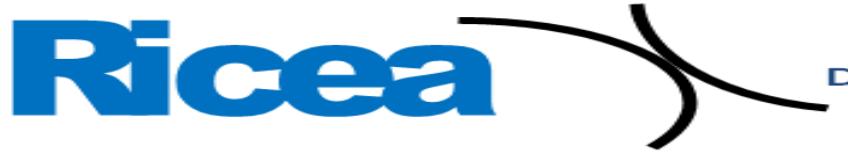

REVISTA IBEROAMERICANA DE CONTADURÍA, ECONOMÍA Y ADMINISTRACIÓN

\section{Introducción}

Los negocios actualmente son desarrollados en un ambiente de constante cambio; un ambiente determinado por factores como la tecnología, las leyes y reformas, crisis financieras y tendencias de consumo que pueden traer consigo nuevas amenazas. Esto es algo con lo que se tiene que lidiar dentro y fuera de la empresa. Por lo que ver oportunidades donde otros no las ven se ha convertido en una de las principales labores del tomador de decisiones. El presente trabajo abarca el proceso de dirección estratégica que apoya la capacidad de toma de decisiones. El objetivo general es diferenciar los elementos de la dirección estratégica mediante las perspectivas que describen al emprendimiento y las características de los modelos de negocio que emergen en un contexto de cambios tecnológicos y mercantil.

Es de suma importancia distinguir cómo las perspectivas sobre el emprendimiento y los modelos de negocio pueden materializar algunas escuelas o tendencias de dirección estratégica (Rodríguez, 2009, p.106 ). Sin duda el emprendimiento es el insumo para proyectar las configuraciones organizacionales a través de los modelos de negocio que se gestan tomando en cuenta la naturaleza de las escuelas de la dirección estratégica.

\section{Etapas de la dirección estratégica}

La dirección estratégica es un proceso que tiene la finalidad de mejorar a la empresa. Y se pueden tener indicadores que ayuden a evaluar dicho proceso: si realmente está ayudando a facilitar el trabajo y a brindar un mejor servicio. Según Cristiana y Anca (2013): "Las principales etapas del proceso de dirección estratégica son la formulación, implementación y evaluación de la estrategia" (p. 1573). Y añaden:

Otra clasificación del proceso de la dirección estratégica que ofrecen Welge y Allea Laham (2008:185-187) y por Borza et al. (2009), consideran las siguientes fases: la planeación de objetivos estratégicos, el análisis estratégico del entorno externo y pronóstico, la elaboración estratégica y su evaluación y, por último, la implementación de la estrategia y su control (Cristiana y Anca, 2013, p. 1576).

Mintzberg, Ahlstrand y Lampel (1999), por su parte, hacen una descripción evolutiva del análisis estratégico, identificando unas escuelas o tendencias caracterizadas por su enfoque limitado, que sometieron a la crítica para indagar sus limitaciones y contribuciones. 
Las 10 escuelas propuestas por Mintzberg (1990) son clasificadas en cuatro grupos: El primer grupo es de naturaleza prescriptiva. Las escuelas que lo conforman se ocupan del modo en que debieran formularse las estrategias: 1) escuela del diseño (década de 1960), estudia la estrategia como un proceso de concepción, como un proceso de diseño informal; 2) escuela de la planificación (1960-1970), se refiere a la estrategia como un proceso formal, como un proceso más independiente y sistemático; 3) escuela del posicionamiento (años 70), hace referencia a la estrategia como un proceso analítico, se concentra en la selección de posiciones dentro del mercado económico.

El segundo grupo es de naturaleza descriptiva. En este caso, consideran aspectos del proceso de creación de estrategias y se interesan en describir su comportamiento ideal: 4) escuela empresarial (años 80), caracterizada por entender a la estrategia como un proceso visionario al describir a este en términos de creación de una visión para el gran líder; 5) escuela cognoscitiva (años 80), estudia la estrategia como un proceso mental, es un proceso de generación conceptual en cabeza de una persona, y utiliza los mensajes de la psicología cognitiva para introducirse en la mente del estratega.

El tercer grupo es de naturaleza grupal, pues amplía más allá de lo individual el proceso de formación de estrategia, extendiéndolo a otros grupos y otros sectores: 6) escuela del aprendizaje (años 80), analiza la estrategia como un proceso emergente; el mundo es complejo para permitir que las estrategias se desarrollen al mismo tiempo como planes o visiones, para ello debe crecer a pequeños pasos a medida que la organización se adapta o aprende de la complejidad. 7) Escuela de poder (década de los 90), caracteriza a la estrategia como un proceso de negociación, en el contexto del conflicto interno y externo de la organización; 8) escuela cultural (años 1990), se refiere a la estrategia como un proceso colectivo, la formación de la estrategia es vista como un proceso colectivo y cooperativo, arraigado a la cultura organizacional; 9) escuela ambiental (años 1990), hace referencia a la estrategia como un proceso reactivo, donde la acción está dirigida hacia el ambiente externo, al comprender las influencias y presiones del entorno. 


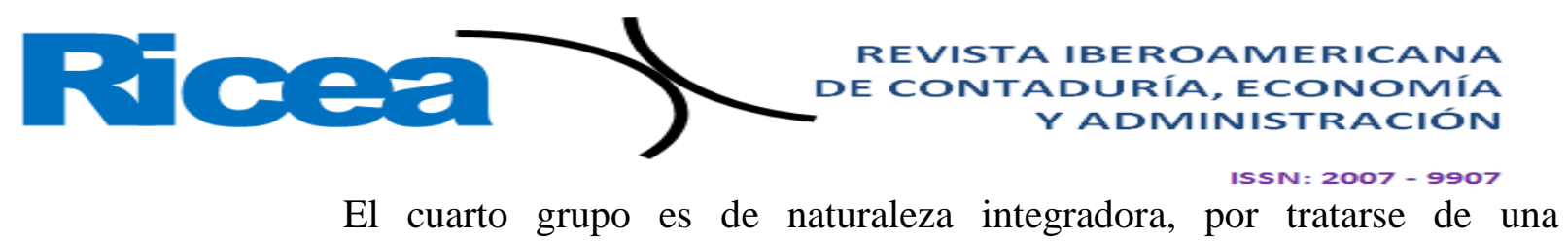
combinación de los grupos y por ende de las escuelas precedentes, y por poseer un carácter transformativo: 10) escuela de configuración (años 2000), estudia la estrategia como un proceso de transformación, se agrupan los diversos elementos del proceso de creación de la estrategia, su contenido, sus estructuras organizacionales y sus contextos en etapas o acontecimientos son ordenados en una secuencia temporal para describir los ciclos vitales de la organización; otro aspecto es el proceso de transformación que incorpora la naturaleza prescriptiva y la práctica de cambio estratégico (Mintzberg, Ahlstrand y Lampel (1999; citado en Rodríguez, 2009, pp. 68-70).

Se puede concluir que las ventajas de aplicar el proceso de la dirección estratégica en las empresas son las siguientes: ofrecer una visión más clara sobre una estrategia, así como sistematizar las decisiones más importantes, lo que facilitaría a los gerentes la mejora del desarrollo de estrategias. La desventaja es que es algo difícil de lograr porque incluye estudios sociológicos, psicológicos y económicos (Cristiana y Anca, 2013, p. 1579).

\section{Concepto de emprendimiento}

Con base en una revisión literaria sobre los aportes para la definición del emprendimiento, se identificaron diferentes propuestas. Una de ellas plantea una clasificación según el pensamiento económico, de las ciencias humanas y de las ciencias de la gestión (Osorio, Gálvez y Murillo, 2010, p. 71). Otra retoma a los clásicos, a los neoclásicos, la escuela de Schumpeter, la escuela austriaca, al igual que otras escuelas de pensamiento, específicamente, según Fonrouge (2002; citado en Rodríguez, 2009, p.102), desde cuatro corrientes: la comportamental, la psicológica (cognitiva), la económica y la de procesos, así como otras aportaciones generales, por ejemplo, el Global Entrepreneurship Monitor (GEM) Colombia Caribe 2010 (Herrera y Montoya, 2013, pp. 20-27). Otra propuesta más busca definir los conceptos de emprendedor y empresario al incorporar solo a los clásicos y a los neoclásicos, así como a la escuela de Schumpeter (Bucardo, Saavedra y Camarena, 2015, pp. 101 y 106).

En relación con el origen del concepto emprendimiento, destaca Richard Cantillon (16801734), "quien es el primero en utilizar el término entrepreneur a fin de diferenciar a un individuo distinto al productor de la economía que recibe salarios y rentas, llamado contratado" (Osorio et al., 2010, p. 71) o "persona que compra los medios de producción a precio, los mezcla y vende a 


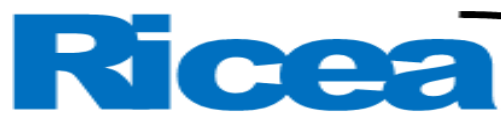

REVISTA IBEROAMERICANA DE CONTADURÍA, ECONOMÍA

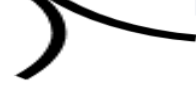

YADMINISTRACIÓN

otro precio no previsible cuando combina los factores" (Gámez, 2008; citado en Herrera y Montoya, 2013, p. 20).

Por su parte, Joseph Alois Schumpeter (1883-1950) señala que "el emprendedor es como alguien innovador más que inventor y que quien financia el proyecto" (Herrera y Montoya, 2013, p. 22). Mientras que Israel Kirzner (1930-) de la escuela austriaca aporta que el emprendedor es "aquel sujeto que descubre en el mundo de conocimiento imperfecto una oportunidad que otros no han percibido" (Kirzner, 1997, 1999; citado en Herrera y Montoya, 2013, p. 22).

Ahora bien, desde las escuelas de pensamiento empresarial, se identifican cuatro perspectivas de pensamiento: la comportamental, la psicológica (cognitiva), la económica y la de procesos (Fonrouge, 2002; citado en Rodríguez, 2009, p. 102). En la tabla 1 se presentan las características de estas cuatro perspectivas. 


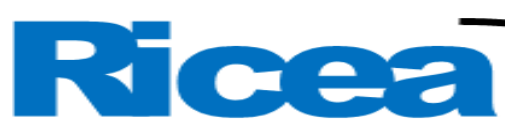

REVISTA IBEROAMERICANA DE CONTADURÍA, ECONOMÍA Y ADMINISTRACIÓN

Tabla 1. Aportes de las escuelas del pensamiento empresarial

\begin{tabular}{|c|c|}
\hline Escuela & Características \\
\hline $\begin{array}{l}\text { Escuela } \\
\text { comportamental }\end{array}$ & $\begin{array}{l}\text { Se define por el conjunto de actividades que se ponen en } \\
\text { marcha para crear una organización. Presentan necesidades de } \\
\text { cumplimiento, de dependencia, un gusto por el riesgo y un } \\
\text { sentimiento por controlar su destino en algunas ocasiones más } \\
\text { enmarcadas que en los administradores (Gartner, 1988; } \\
\text { Rodríguez y Jiménez, 2005; ambos citados en Herrera y } \\
\text { Montoya, 2013, p. 24). }\end{array}$ \\
\hline $\begin{array}{l}\text { Escuela psicológica con } \\
\text { las corrientes } \\
\text { personalistas } \\
\text { cognitivas }\end{array}$ & $\begin{array}{l}\text { Se define por un cierto número de atributos psicológicos } \\
\text { descritos tanto por la personalidad como por los procesos } \\
\text { cognitivos activados por las circunstancias (Shaver y Scott } \\
\text { 1991; Rodríguez y Jiménez, 2005; ambos citados en Herrera y } \\
\text { Montoya, 2013, p. 24). }\end{array}$ \\
\hline Escuela económica & $\begin{array}{l}\text { Se especializa en la toma de decisiones pensadas y relativas a } \\
\text { la coordinación de recursos escasos (Casson, 1991; citado en } \\
\text { Herrera y Montoya, 2013, p. 24). }\end{array}$ \\
\hline Escuela de procesos & $\begin{array}{l}\text { Es aquella que desarrolla oportunidades y crea una } \\
\text { organización para explotarlas (Bygrave y Hofer, 1991; } \\
\text { Rodríguez y Jiménez, 2005; ambos citados en Herrera y } \\
\text { Montoya, 2013, p. 24). }\end{array}$ \\
\hline
\end{tabular}

Fuente: Fonrouge (2002; citado en Herrera y Montoya, 2013, p. 24)

Por otro lado, el GEM Colombia Caribe 2010 utiliza tres tipologías para los emprendedores, de acuerdo con la Nueva Actividad Empresarial (TEA): emprendedores potenciales, empresarios nacientes y nuevos empresarios. Los emprendedores se caracterizan en su gran mayoría por ser hombres jóvenes de 25 a 34 años, con educación secundaria completa, ingresos bajos; reciben información o experiencia de una persona cercana; participan en redes de emprendimiento; son innovadores, atentos a las oportunidades, tomadores de riesgos y que consideran que la creación de empresas es la "carrera" ideal (Herrera y Montoya, 2013, p. 26). 

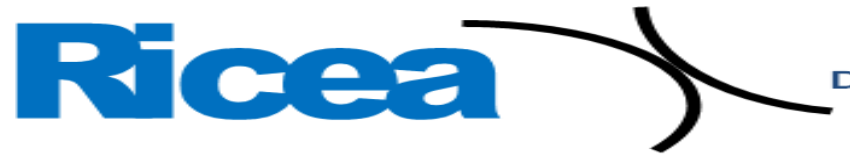

REVISTA IBEROAMERICANA DE CONTADURÍA, ECONOMÍA Y ADMINISTRACIÓN

En lo que se refiere al concepto empresario, se contemplan a tres autores clásicos como son Adam Smith (1723-1790), quien define lo define "como propietario de los medios de producción y patrón o maestro del trabajo" (Bucardo et al., 2015, p.106); así como a Richard Cantillon (1680-1734) y Jean-Baptiste Say (1767-1832), quienes señalan que se trata de un "agente u hombre de negocios que compra los medios de producción a ciertos precios y los combina en un producto que venderá a un precio incierto en el momento de comprometer sus costes" (Bucardo et al., 2015, p.106).

También se retoma a dos neoclásicos. Según Alfred Marshall (1842-1924), “el empresario como cuarto factor productivo, con el que se aporta el conocimiento a través de la organización y cuya función es la dirección de los negocios" (Bucardo et al., 2015, p.106 ). Frank Knight (18851897), por su parte, indica que "el empresario como figura que asume el riesgo de la actividad económica al anticipar el producto nacional, creando y asegurando rentas, por lo que el beneficio empresarial o renta residual es el beneficio es la remuneración de dicho riesgo" (Bucardo et al., 2015, p.106). Otra escuela más es la representada por Joseph Schumpeter (1883-1950), quien identifica al "empresario como innovador o agente principal del desarrollo económico capitalista, consecuencia del cambio tecnológico. Liderazgo innovador que justifica su beneficio como renta residual de la economía” (Bucardo et al., 2015, p. 106; Herrera y Montoya, 2013, pp. 20-22).

Como se puede observar, la definición del emprendedor y del empresario no es la misma, aunque muchas veces se suele confundir ambos términos y utilizar como sinónimos. Se infiere, más bien, que el emprendedor es el innovador y el empresario es el dueño de los medios de producción. A continuación, se expone una conclusión respecto a la diferenciación de estos conceptos:

El emprendedor es quien utiliza el ingenio, la audacia, el entusiasmo, la ilusión y la insatisfacción laboral para realizar un cambio a través de la innovación de las ideas, los productos o los procesos, sin que esto implique necesariamente su participación en la creación o dirección de las empresas. Por otra parte, el empresario es quien invierte en la empresa capital propio o ajeno y la dirige con la finalidad de obtener un rendimiento (Bucardo et al., 2015, p. 106). 


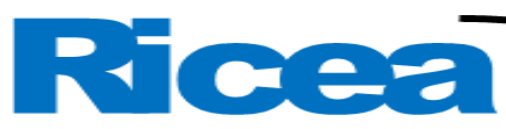

REVISTA IBEROAMERICANA DE CONTADURÍA, ECONOMÍA Y ADMINISTRACIÓN

\section{Concepto y características del modelo de negocios}

Con respecto al modelo de negocio, desde una perspectiva de procesos, existen las definiciones vertidas en la tabla 2.

Tabla 2. Definiciones del concepto modelo de negocio

\begin{tabular}{|c|c|}
\hline Autores & Definición \\
\hline $\begin{array}{l}\text { Zott y Amit (2009, p. 110; } \\
\text { citados en Palacios y Duque, } \\
\text { 2011, p. 25) }\end{array}$ & $\begin{array}{l}\text { "Forma en que una empresa 'hace negocios' con } \\
\text { sus clientes, socios y proveedores; es decir, se } \\
\text { trata del sistema de actividades específicas que la } \\
\text { empresa focal o sus socios llevan a cabo para } \\
\text { satisfacer las necesidades percibidas en el } \\
\text { mercado; cómo esas actividades están } \\
\text { relacionadas entre sí, y quién lleva a cabo esas } \\
\text { actividades". }\end{array}$ \\
\hline $\begin{array}{l}\text { Osterwalder y Pigneur } \\
(2009 \text {, p. 14; citados en } \\
\text { Palacios y Duque, 2011, p. } \\
\text { 25) }\end{array}$ & $\begin{array}{l}\text { "Un modelo de negocio describe la lógica de } \\
\text { cómo una organización crea, entrega, y captura } \\
\text { valor". }\end{array}$ \\
\hline $\begin{array}{l}\text { Svejenova, Planellas y Vives } \\
(2010 \text {, p. 409; citados en } \\
\text { Palacios y Duque, 2011, p. } \\
\text { 25) }\end{array}$ & $\begin{array}{l}\text { "Conjunto de actividades, organización y } \\
\text { recursos estratégicos que transforman la } \\
\text { orientación establecida por la empresa en una } \\
\text { proposición de valor distintiva, permitiendo a la } \\
\text { misma crear y capturar valor". }\end{array}$ \\
\hline
\end{tabular}

Fuente: Palacios y Duque (2011, p. 25)

Teniendo en cuenta las definiciones antes expuestas sobre el concepto modelo de negocio, se puede inferir que se trata de identificar "el componente de capacidades y procesos cuyos aspectos estudiados son procesos internos, recursos y capacidades, así como las fuentes de ventajas competitivas que respondan a la pregunta clave: cuál es la fuente interna de la ventaja competitiva" (Cervilla y Puente, 2013, p. 294) o “cuáles son los recursos y procesos necesarios para la formulación de un modelo de negocio para centros de productividad?" (Palacios y Duque, 2011, p. 31). 


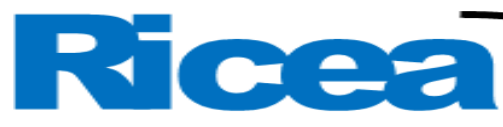

REVISTA IBEROAMERICANA DE CONTADURÍA, ECONOMÍA Y ADMINISTRACIÓN

En lo concerniente al diseño del modelo de negocio, se abordan las propuestas incluidas en la tabla 3.

Tabla 3. Propuestas para diseñar un modelo de negocio

\begin{tabular}{|c|c|}
\hline Autores & Propuesta \\
\hline $\begin{array}{l}\text { Zott y Amit (2009; citados en } \\
\text { Palacios y Duque, 2011, p. 27) }\end{array}$ & $\begin{array}{l}\text { "Las preguntas que los directivos necesitan } \\
\text { plantearse para la innovación del modelo de } \\
\text { negocio son las siguientes: ¿Cuál es el objetivo } \\
\text { del nuevo modelo de negocio? ¿Qué nuevas } \\
\text { actividades son necesarias para satisfacer las } \\
\text { necesidades percibidas? ¿Cómo podrían esas } \\
\text { actividades estar relacionadas entre sí de } \\
\text { formas novedosas? ¿Quién debería realizar } \\
\text { cada una de las actividades que forman parte } \\
\text { del modelo de negocio (por ejemplo, la } \\
\text { empresa focal o un socio), y qué disposiciones } \\
\text { novedosas en materia de gestión podrían } \\
\text { habilitar esa estructura? ¿Cómo se crea el valor } \\
\text { a través del nuevo modelo de negocio para } \\
\text { cada una de las partes involucradas? y ¿ué } \\
\text { modelo de ingresos de la empresa focal } \\
\text { permitirá a la compañía apropiarse de parte del } \\
\text { valor creado a partir del nuevo modelo de } \\
\text { negocio". }\end{array}$ \\
\hline $\begin{array}{l}\text { Vives y Svejenova (2009; citados } \\
\text { Palacios y Duque, 2011, p. 28) }\end{array}$ & $\begin{array}{l}\text { "Determinan las preguntas claves para la } \\
\text { definición de un modelo de negocio así: ¿Cuál } \\
\text { es la necesidad y comportamiento del cliente y, } \\
\text { por lo tanto, ¿cómo va a definir su mercado? } \\
\text { ¿Qué va a ofrecer, a qué precio, y cómo esta } \\
\text { oferta es diferencial frente a otras existentes en } \\
\text { el mercado? ¿Cómo lo va a organizar? ¿Cómo }\end{array}$ \\
\hline
\end{tabular}




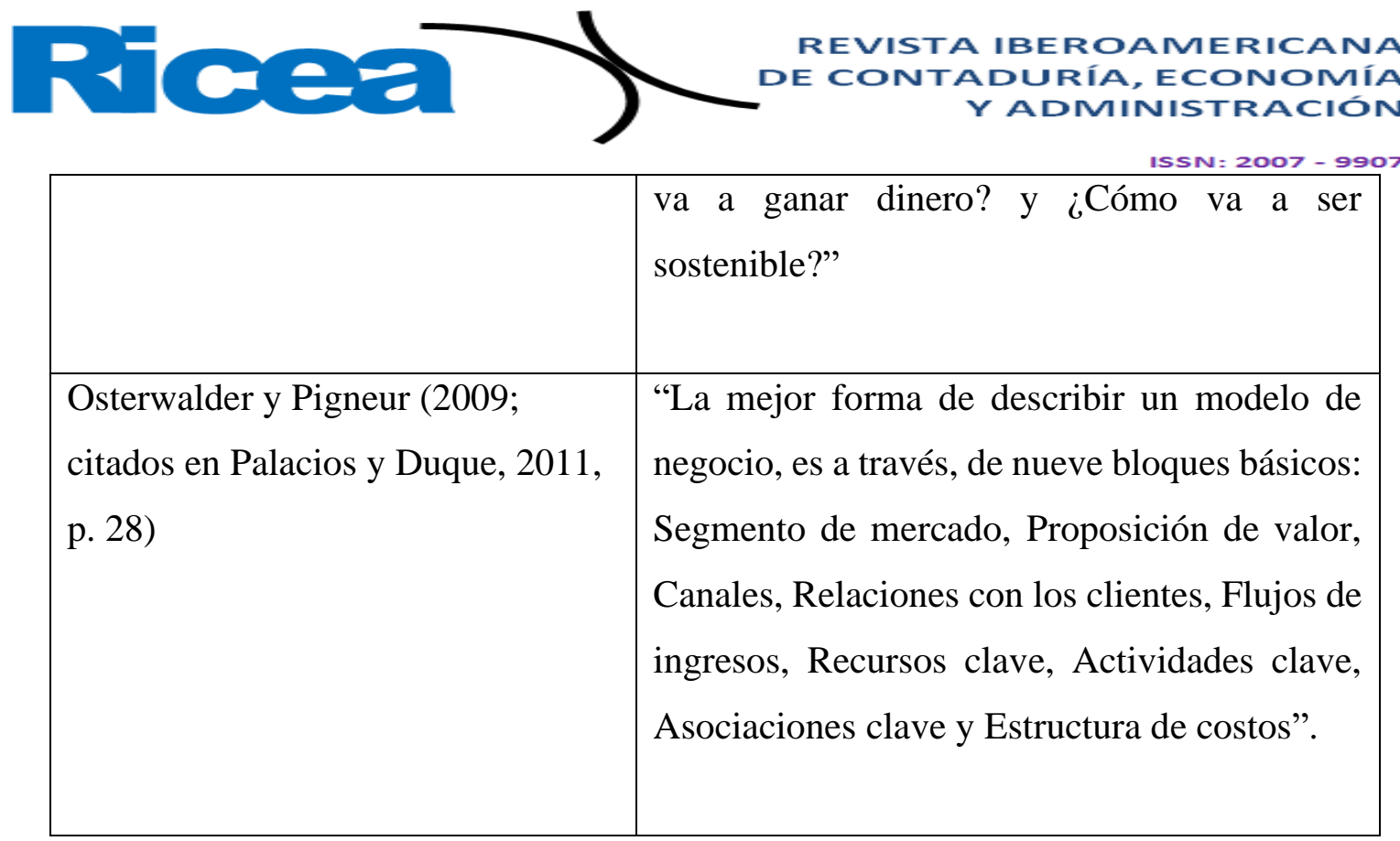

Fuente: Palacios y Duque (2011, pp. 27-28)

Como resultado de la revisión de las anteriores propuestas, es importante considerar que "la propuesta de valor actualmente es el componente esencial y vital en la creación del modelo de negocios (Beckmezi, 2013; Comes, 2008; ambos citados en Mejía y Sánchez, 2014, p. 113). Tomando en cuenta que "más del $90 \%$ de todas las innovaciones en los modelos de negocios (IMN) son recombinación de modelos, ideas y conceptos" (Fleish, 2012; citado en Mejía y Sánchez, 2014, p. 114). Por lo tanto, el avance en la tecnología, la regulación y el descubrimiento de nuevos nichos de mercado, entre otros, fuerzan a las firmas a hacer una revisión permanente de sus modelos de negocio (Mejía y Sánchez, 2014, p. 124).

\section{Estudios recientes sobre emprendimiento y modelos de negocio}

El modelo de negocio sirve como una herramienta para dar seguimiento a la evolución de la empresa de nueva creación. Al respecto, Batista, Bolívar y Medina (2016) estudiaron sobre las causas del fenómeno del emprendimiento y los elementos que rodean al mismo, la actuación del emprendedor y de su empresa durante la fase de inicio, así como su manera de interpretar el entorno en el que se encuentra y cómo esto afecta en la toma de decisiones consecuentes.

Estos autores presentaron una propuesta para la monitorización del proceso emprendedor a través del modelo de negocio como una herramienta de prospección y estudio del fenómeno del emprendedor en la fase incipiente. El monitoreo se llevó a cabo realizando el siguiente protocolo: seguimiento continuo de iniciativas empresariales, análisis de las empresas creadas y seguimiento de su actividad, elaboración de los casos para su estudio, donde se detectó la empresa objeto de análisis: empresa mayor a 42 meses de actividad, no era de base tecnológica ni internacionalizada 


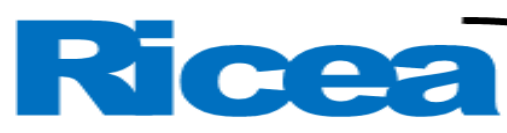

REVISTA IBEROAMERICANA DE CONTADURÍA, ECONOMÍA Y ADMINISTRACIÓN

en su origen y el patrón de crecimiento de la empresa no encajaba en los modelos tradicionales (Batista et al., 2016, pp. 88 y 96).

En la empresa objeto de análisis mencionada, asimismo, durante el inicio del negocio se pudo apreciar la inmadurez en el modelo de negocio del emprendimiento por la carencia de un vínculo a socios externos. A los 25 meses de su creación, se realizó un giro en la propuesta de valor, y agregaron más servicios y ampliaron la segmentación de mercado. Finalmente, a los 42 meses de estar activa la empresa, logró posicionarse con diversas ventajas de competencia debido a sus relaciones externas y su visión. Al momento del cierre del estudio, se tenía el objetivo de expandirse a nivel internacional (Batista et al., 2016, pp. 90-93).

Como ya se mencionó líneas atrás, se suele definir a un emprendedor como aquella persona que ve las oportunidades y toma el riesgo de crear nuevos productos o procesos con la finalidad de crear riqueza. Y precisamente uno de los temas más estudiados es el de los atributos que constituyen a un emprendedor; mientras que se ha dejado de lado el estudio de los modelos de negocio utilizados por ellos, sobre todos los delineados por la base de la pirámide (BDP). Detectando este patrón, Cervilla y Puente (2013) seleccionaron a diez emprendimientos dirigidos por la BDP con más de tres años de vigencia y analizaron sus modelos de negocio.

Como parte de los resultados tuvieron los siguientes elementos en común: el negocio fue iniciado por necesidad, la idea del negocio surgió mientras trabajaban para otra empresa o como algo extra con poca ganancia, el nivel promedio educativo de los emprendedores es medio, sin capacitación o estudios sobre el emprendimiento, se percibe al emprendimiento como un medio de independencia económica con el cual se puede obtener mayores ingresos a su propio ritmo de trabajo, el crecimiento empresarial se hace con base en cubrir sus necesidades y obtener una calidad de vida apropiada, evitando crecer en mayor medida para no enfrentarse a nuevos retos, generalmente ellos mismos manejan el negocio o tienen la ayuda de un familiar y rara vez alguien de fuera, sus negocios están cerca de la zona donde viven, no creen necesario llevar algún tipo de capacitación o curso para administrar su negocio y no son innovadores y reconocen que el apoyo de los bancos para la apertura de su negocio fue más allá del dinero (Cervilla y Puente, 2013, pp. 295-298).

Finalmente, el modelo de negocio aporta una conexión natural entre la formulación y la implantación de la estrategia. Es decir: la separación tan poco natural entre el pensar y el hacer desaparece cuando uno utiliza el modelo de negocio como reflejo y proyección de la estrategia. Por todas estas razones, se cree que el concepto de modelo de negocio tiene una gran capacidad para integrar aspectos que muchas veces artificialmente hemos separado en el campo de la 

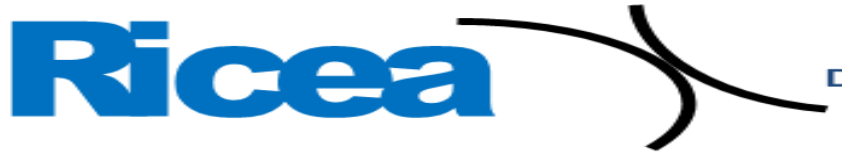

REVISTA IBEROAMERICANA DE CONTADURÍA, ECONOMÍA Y ADMINISTRACIÓN

estrategia y por ello constituye ese eslabón perdido en la dirección estratégica (Ricart, 2009, p. 22).

En suma, el principal objetivo de un modelo de negocios es el de generar valor en la empresa con base en la innovación, lo cual, a partir de diversas estrategias, ayuda a la empresa a crear una ventaja competitiva.

\section{Metodología}

El método de esta investigación comprendió una revisión de diversas fuentes de literatura realizada entre los meses de enero a octubre de 2018 en distintas bases de datos, a saber: Scielo, Redalyc, Researchgate y Dialnet. Cabe aclarar que se consideraron los artículos que incluían las palabras claves de la búsqueda, acorde a un periodo del 2014 a la fecha; y en relación con el idioma, se eligieron aquellos que fueran en español e inglés.

\section{Resultados y discusión}

El objetivo general de la presente investigación es diferenciar los elementos de la dirección estratégica mediante las perspectivas que describen al emprendimiento y las características de los modelos de negocios que emergen en un contexto de cambios tecnológicos y los mercados.

En lo que se refiere a la dirección estratégica, tiene la finalidad de mejorar a la empresa utilizando metodologías para el diseño de un proceso; sin embargo, la intención de la dirección estratégica es con fines de autosuperar a la organización. De tal manera que la dirección estratégica no solo busca un crecimiento empresarial económico, sino un reconocimiento social que permita implementar las revoluciones industriales donde se manifiesta el emprendimiento y se materializa el modelo de negocio.

Con respecto a los elementos de la dirección estratégica que se pueden diferenciar en las perspectivas sobre el emprendimiento, existen dos escuelas (empresarial y cognitiva) que son de naturaleza descriptiva, dado que el emprendedor considera "el proceso de creación de estrategias y se interesa en describir su comportamiento ideal" (Rodríguez, 2009, p. 69), y una más de procesos, que es una de las escuelas de pensamiento empresarial, según Fonrouge (2002; citado en Herrera y Montoya, 2013), que muestra "cómo se desarrollan las oportunidades a fin de crear una organización para ser aprovechadas" (p. 25). Por esta razón, el emprendedor es quien implementa estrategias para adaptarse al entorno con la finalidad de lograr adentrarse al mercado. 

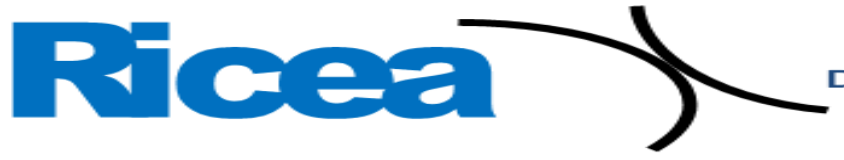

REVISTA IBEROAMERICANA DE CONTADURÍA, ECONOMÍA Y ADMINISTRACIÓN

En cambio, el empresario “amplía más allá de lo individual el proceso de formación de estrategia, extendiéndolo a otros grupos y otros actores (Herrera y Montoya, 2013, p. 69). En tal sentido, se puede retomar a Alfred Marshall (Bucardo et al., 2015), "que se ocupa de medir el papel del empresario en el proceso productivo como un factor adicional a los tres factores productivos clásicos de tierra, trabajo y capital", o bien "como cuarto factor productivo, con el que se aporta el conocimiento a través de la organización y cuya función es la dirección de negocios" (pp. 105-106).

En las reflexiones anteriores respecto a la distinción de las escuelas de dirección estratégica y en la diferenciación del emprendedor respecto al empresario, hay que tener en cuenta la naturaleza descriptiva o grupal de las respectivas escuelas.

Con base en la misma forma de análisis, se contempla en el concepto y características del modelo de negocios a "las estrategias de naturaleza prescriptiva que comprende tres escuelas (diseño, planificación y posicionamiento) que se ocupan del modo en que se debieran formularse las estrategias" (Rodríguez, 2009, p. 69). De todo esto se desprende que el modelo de negocio es un reflejo de una de las etapas de la dirección estratégica que es la formulación o la elaboración de la estrategia, que es necesaria para la mejora de la empresa.

Además, se vislumbra que en el diseño del modelo de negocios es necesario reconocer que la clasificación de las 10 escuelas de dirección estratégica mencionada anteriormente aborda una propuesta implícita que puede aportar una secuencia instruccional prescriptiva, descriptiva, grupal e integradora que permita destacar las etapas de la propia dirección estratégica en la creación del modelo de negocios que sistematice el proceso de emprendimiento con un direccionamiento estratégico. 

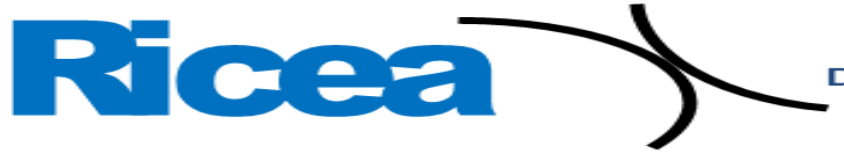

REVISTA IBEROAMERICANA DE CONTADURÍA, ECONOMÍA Y ADMINISTRACIÓN

\section{Conclusiones}

Con base en el objetivo general de la presente investigación, se puede vislumbrar que el proceso de emprendimiento surge a raíz de una oportunidad en el entorno o por necesidad del propio emprendedor que se configura en un modelo de negocio centrado en crear o generar un valor que se materializa en una ventaja competitiva.

Cabe aclarar que el modelo de negocios describe la manera de negociar con el cliente con el objetivo de generar ingresos. Además, es una herramienta para dar seguimiento a la evolución de la empresa. En fin, tomando en cuenta lo anterior, así como la caracterización del emprendedor como el creador de la estrategia y el modelo de negocio como una forma de indagar o recopilar información para entender la oportunidad o la necesidad de emprender, se debe destacar que, en conjunto, buscan el mejoramiento, desde cualquier perspectiva o escuela de pensamiento estratégico, para la evolución de la organización, industria, negocio o empresa.

En suma, las estrategias son las causas de la relación entre emprendimiento y la innovación de los modelos de negocio, pues la etapa de gestación del emprendimiento permite vislumbrar el tipo de modelo de negocio que se pretende establecer con base en la naturaleza de las tendencias de dirección estratégica, lo cual ofrece un marco de referencia clave para comprender la evolución del pensamiento estratégico.

\section{Agradecimientos}

En lo particular, se agradece a la Dra. María Guadalupe Veytia Bucheli de la Universidad Autónoma del Estado de Hidalgo por su asesoría profesional y personal para realizar las distintas actividades del Postdoctorado de Administración de Negocios en Línea.

Además, reconocer el apoyo brindado en la revisión y análisis de la literatura por parte de Dania Sarahi Fernández Mercado, estudiante de la Maestría en Administración, y de Gerardo Alexis Ramírez Murillo, estudiante de la Licenciatura en Derecho, ambos de la Universidad Autónoma de Ciudad Juárez. 


\section{Referencias}

Batista, R., Bolívar, A. y Medina, P. (2016). Monitorización del proceso emprendedor a tráves del modelo de negocio. Revista Innovar, 26(61), 83-101.

Bucardo, A., Saavedra, M. L. y Camarena, M. E. (2015). Hacia una comprensión de los conceptos de emprendedores y empresarios. Suma de Negocios, 6(13), 98-107.

Cervilla, M. A. y Puente, R. (2013). Modelos de negocio de emprendimientos por y para la base de la pirámide. Revista de Ciencias Sociales (Ve), 19(2), 289-308.

Cristiana, P. Z. and Anca, B. (2013). New perspectives on strategic management process. Annals of Faculty of Economics and Business Administration, 1(1), 1573-1580.

García, M. (2016). Modelos de negocio que triunfan: reflexiones sobre innovación y start-ups. Sociedad y Utopía. Revista de Ciencias Sociales, (47), 226-238.

Herrera, C. E. y Montoya, L. A. (2013). El emprendedor: una aproximación a su definición y caracterización. Punto de Vista, 4(7), 7-30.

Mejía, J. y Sánchez, J. (2014). Factores determinantes de la innovación del modelo de negocios en la creación de ventaja competitiva. Revista del Centro de Investigación. Universidad La Salle, 11(42), 105-128.

Mintzberg, H. (1999). Safari Strategy. New York, United States: The Free Press.

Osorio, F. F., Gálvez, E. J. y Murillo, G. (2010). La estrategia y el emprendedor: diversas perspectivas para el análisis. Cuadernos de Administración, (43), 65-80.

Palacios, M. y Duque, E. (2011). Modelos de negocio: propuesta de un marco conceptual para centro de productividad. Administración \& Desarrollo, 39(53), 23-34.

Pulido, B. (2010). Teoría de los recursos y capacidades: El foco estratégico centrado en el interior de la organización. Sotavento M.B.A., (15), 54-61.

Ricart, J. E. (2009). Modelo de negocio: El eslabón perdido en la dirección estratégica. Universia Business Review, (23), 12-22.

Rodríguez, A. (2009). Nuevas perspectivas para entender el emprendimiento empresarial. Pensamiento \& Gestión, (26), 94-119. 
Rodríguez Ramírez, A. (2009). Influencia del Entorno en el Desarrollo del Direccionamiento

Estratégico . Revista Cuadernos de Administración (Cali-Colombia), 68-70.

\begin{tabular}{|l|l|}
\hline Rol de Contribución & Autor (es) \\
\hline Conceptualización & Gabriela Velasco Rodríguez (principal) \\
\hline Metodología & Gabriela Velasco Rodríguez (principal) \\
\hline Software & NA \\
\hline Validación & NA \\
\hline Análisis Formal & NA \\
\hline Investigación & NA \\
\hline Recursos & NA \\
\hline Curación de datos & NA \\
\hline $\begin{array}{l}\text { Escritura - Preparación del } \\
\text { borrador original }\end{array}$ & Gabriela Velasco Rodríguez (principal) \\
\hline $\begin{array}{l}\text { Escritura - Revisión y } \\
\text { edición }\end{array}$ & $\begin{array}{l}\text { Gabriela Velasco Rodríguez (principal) } \\
\text { María Guadalupe Veytia Bucheli (apoya) }\end{array}$ \\
\hline Visualización & Gabriela Velasco Rodríguez (principal) \\
María Guadalupe Veytia Bucheli (apoya)
\end{tabular}

